\title{
Gestión de Cobranza: un abordaje teórico desde el ámbito financiero
}

\section{Collection Management: a theoretical approach from the financial field}

Leydy Díaz

myleidy2005@gmail.com

Código ORCID: 0000-0002-2609-1698

Universidad Peruana Unión - Perú

\author{
Daibis Yesica Flores Enríquez \\ yesicaflores03@gmail.com \\ Código ORCID: 0000-0002-4103-7489
}

Universidad Peruana Unión - Perú

Artículo recibido abril 2019 / Arbitrado en mayo 2019 / Publicado en julio 2019

RESUMEN
El objetivo de la revisión tiene por finalidad determinar de qué manera una buena gestión de cobranza impacta favorablemente a las empresas, asimismo abordar de manera teórica la importancia de la gestión de cobranza a partir de hechos que suceden en los diferentes ámbitos comerciales de dónde surge como respuesta al sistema financiero de una empresa, para dar recursos que puedan aumentar la liquidez mediante una adecuada gestión de cobranza. Es así pues que en la búsqueda se detectó que las principales causas del retraso en las cuentas por cobrar, es la ausencia de adecuadas políticas de cobranzas para otorgar el plazo de días de pago a los clientes y la falta de un adecuado compromiso del personal con la organización. En este sentido, la gestión de las cuentas por cobrar está dirigido a incrementar la liquidez de la empresa, siendo así necesario las políticas de cobranza, que permitirá ver en qué situación se encuentra la empresa frente a sus obligaciones financieras. En ese sentido el análisis del estado que presentaba la empresa se ha encontrado la falta de políticas definidas, sobre todo para los créditos a empresas extranjeras y el personal no está capacitado para las funciones que el área necesita.

Palabras clave: Gestión de cobranza; Cuentas por cobrar; políticas de cobranza, liquidez

The purpose of this review is to determine how good collection management favorably impacts companies, as well as theoretically addressing the importance of collection management based on events that occur in the different commercial areas from which it arises as response to the financial system of a company, to provide resources that can increase liquidity through good collection management. Thus, in the search it was found that the main causes of the delay in the accounts receivable, is the absence of adequate collection policies to grant the period of days of payment to the clients and the lack of an adequate commitment of the staff with the organization. In this sense, the management of the accounts receivable are aimed at increasing the liquidity of the company, thus being necessary the collection policies, which will allow to see in what situation the company is in front of its financial obligations. In this sense, the analysis of the state presented by the company has found the lack of defined policies, especially for loans to foreign companies and the staff is not trained for the functions that the area needs.

Key words: Collection management; Accounts receivable; collection policies, liquidity 


\section{INTRODUCCIÓN}

Hoy en día, las empresas vienen brindando sus servicios de una manera que les permite generar más rentabilidad y al mismo tiempo satisfacer a los clientes que perciben sus servicios. Es por eso, que brindan las facilidades necesarias, pero al mismo tiempo se han ido incrementando los clientes deudores o morosos, convirtiéndose en una problemática para la empresa afectando el patrimonio, lo cual es primordial para el progreso y desarrollo de la misma, ya que la demora de los pagos genera problemas de liquidez en las empresas. En tal sentido, Ruiz y Lopez (2017) indica que una adecuada gestión se cobranza llevada eficientemente por los gestores de cobranza tendrá el resultado esperado que es la disminución de la cartera y en el peor escenario la contención de la morosidad.

Además, Castillo \& Córdova (2016) indican que la Gestión de Créditos y Cobranza se ve afectada por la falta de un sistema de procedimientos que permitan un buen control para la recaudación de pagos de sus clientes, lo que genera un alto nivel de morosidad y a la vez disminuye los ingresos de efectivo a la empresa. Además, Carrera (2017) indica que en la actualidad las empresas se ven necesitadas de una adecuada gestión de cuentas por cobrar, a fin de tomar decisiones apropiadas y acertadas en favor del desarrollo de la institución. Adicionalmente, Díaz y Ramos (2018) mencionan la importancia que tiene en el entorno empresarial la mejora del sistema de cobros de una forma más oportuna, ya que la excesiva demora afecta la liquidez de la empresa y ésta perjudica a la capacidad de pago.

Santillán (2016), en su estudio menciona que las políticas y procedimientos de las cuentas por cobrar que las empresas tienen, deben tener un control adecuado, utilizando técnicas de cobranza eficaces, así como no dar créditos a largo plazo, son un riesgo para la empresa, además de conocer el impacto que tienen las cuentas incobrables lo cual desequilibra financieramente las operaciones de la empresa. Del mismo modo Flores y Naval (2016) realizaron un estudio respecto a la rentabilidad y las cuentas por cobrar obteniendo como resultados que los indicadores de gestión de actividad y rentabilidad Financiera de las organizaciones tienen correlación, concluyendo que la rotación de los inventarios y de las cuentas por cobrar inciden positivamente en la rentabilidad de las organizaciones empresariales.

Además, Balarezo (2019) en sus resultados manifiesta que algunas empresas cubren sus obligaciones, pero esto les causa limitaciones de efectivo. Por otro lado, los proveedores, al querer cerrar el mes y cumplir su meta, ofrecen plazos para cobrar sus créditos otorgados que luego no cumplen, exigiendo el pago en fechas antes a la negociada, lo cual genera problemas para el despacho de la siguiente venta, causando retrasos y en muchas ocasiones imposibilita la venta.

Así mismo, dentro de las empresas, la falta de organización, respecto a las condiciones para otorgar créditos a los clientes, genera confusión en la gestión de cobranzas. Se hace necesario e importante aplicar el indicador financiero de liquidez al momento de evaluar la gestión de un periodo, de tal modo la empresa pueda tener la capacidad de proponer mejoras y previsiones para los períodos siguientes.

Según las investigaciones realizadas y consideradas por este estudio, se observa que el conocimiento de los resultados que puedan darse al analizar la influencia de gestión de 
cobranza, son de vital importancia para garantizar a las empresas el retorno económico a corto plazo, de tal modo que se puedan cumplir con las obligaciones y asimismo reducir los índices en la morosidad, tal como se evidencia en la .

Tabla 1.

Tabla 1. Evidencia empírica sobre Gestión de Cobranza

\begin{tabular}{|c|c|c|c|}
\hline Autor(es) & $\begin{array}{c}\text { Tema } \\
\text { (Gestión de cobranza y } \\
\text { morosidad) }\end{array}$ & Objetivo & Principal resultado \\
\hline $\begin{array}{l}\text { Gallegos, } \\
\text { Galarreta, } \\
\text { Ruiz y } \\
\text { Gutiérrez } \\
(2016)\end{array}$ & $\begin{array}{l}\text { Estudio de Métodos para } \\
\text { disminuir el Îndice de } \\
\text { Morosidad. Art. }\end{array}$ & $\begin{array}{l}\text { Identificar e implementar } \\
\text { métodos de cobranza. }\end{array}$ & $\begin{array}{l}\text { Los métodos en el proceso } \\
\text { de cobranza lograron } \\
\text { disminuir el } 5 \% \text { de la } \\
\text { morosidad. }\end{array}$ \\
\hline Ruiz (2017). & $\begin{array}{l}\text { Diseño de un modelo } \\
\text { matemático para la calificación } \\
\text { de clientes morosos en } \\
\text { una entidad comercial } \\
\text { mediante las metodologías de } \\
\text { árboles de decisión, } \\
\text { análisis discriminante y } \\
\text { regresión logística. Art. }\end{array}$ & $\begin{array}{l}\text { Priorizar la gestión de } \\
\text { cobro en edades } \\
\text { tempranas de morosidad } \\
\text { es de vital importancia en } \\
\text { las empresas para lograr } \\
\text { la recuperación de } \\
\text { créditos }\end{array}$ & $\begin{array}{l}\text { El modelo matemático } \\
\text { logró optimizar la prioridad } \\
\text { de cobro de una cartera } \\
\text { con morosidad temprana }\end{array}$ \\
\hline $\begin{array}{l}\text { Castillo y } \\
\text { Córdova } \\
\text { (2017) }\end{array}$ & $\begin{array}{l}\text { La Gestión de Créditos y } \\
\text { Cobranzas y su influencia en el } \\
\text { nivel de Morosidad }\end{array}$ & $\begin{array}{l}\text { Conocer si la Gestión de } \\
\text { Créditos y cobranzas } \\
\text { influye en el nivel de } \\
\text { morosidad. }\end{array}$ & $\begin{array}{l}\text { La Gestión de Créditos y } \\
\text { Cobranzas influyó en un } \\
32 \% \text { el desempeño } \\
\text { eficiente en la reducción } \\
\text { del nivel de morosidad }\end{array}$ \\
\hline $\begin{array}{l}\text { Guevara y } \\
\text { Upiachihuia } \\
\text { (2019) }\end{array}$ & $\begin{array}{l}\text { Gestión de cobranza y su } \\
\text { incidencia en la morosidad }\end{array}$ & $\begin{array}{l}\text { Determinar la incidencia } \\
\text { de la gestión de cobranza } \\
\text { en la morosidad }\end{array}$ & $\begin{array}{l}\text { El empleo de gestión de } \\
\text { cobranza permitió } \\
\text { disminuir índice de } \\
\text { morosidad en un } 21 \% \text { y un } \\
\text { índice de cobertura de un } \\
40 \%\end{array}$ \\
\hline $\begin{array}{l}\text { Miranda } \\
\text { (2018) }\end{array}$ & $\begin{array}{l}\text { Estrategias de cobranza y } \\
\text { morosidad }\end{array}$ & $\begin{array}{l}\text { Determinar la relación } \\
\text { entre estrategias de } \\
\text { cobranza y la morosidad }\end{array}$ & $\begin{array}{l}\text { Se concluyó que existe una } \\
\text { relación significativa entre } \\
\text { las variables estrategias de } \\
\text { cobranza y la morosidad }\end{array}$ \\
\hline
\end{tabular}




\begin{tabular}{|c|c|c|c|}
\hline Autor(es) & $\begin{array}{c}\text { Tema } \\
\text { (Gestión de cobranza y } \\
\text { morosidad) }\end{array}$ & Objetivo & Principal resultado \\
\hline Bastra (2018) & $\begin{array}{l}\text { Propuesta para disminuir el } \\
\text { índice de morosidad según el } \\
\text { modelo de gestión amistosa } \\
\text { de cobranzas en el Colegio } \\
\text { Adventista "El Porvenir" }\end{array}$ & $\begin{array}{l}\text { Elaborar una propuesta } \\
\text { para disminuir el índice } \\
\text { de morosidad, según el } \\
\text { modelo de gestión } \\
\text { amistosa de cobranzas }\end{array}$ & $\begin{array}{l}\text { La propuesta en mención } \\
\text { permitirá disminuir el índice } \\
\text { de morosidad a un } 5 \% \text { en } \\
\text { promedio. }\end{array}$ \\
\hline $\begin{array}{l}\text { Ortiz, } \\
\text { Nolazco y } \\
\text { Carhuancho } \\
(2020)\end{array}$ & $\begin{array}{l}\text { Las Cuentas por cobrar en una } \\
\text { Empresa de Servicios, Lima } \\
2018 \text { Art. }\end{array}$ & $\begin{array}{l}\text { Diagnosticar la situación } \\
\text { de las cuentas por cobrar } \\
\text { y su consecuencia en los } \\
\text { estados financieros }\end{array}$ & $\begin{array}{l}\text { Se determinó que la } \\
\text { cuentas por cobrar } \\
\text { representan en relación al } \\
\text { total de activos el } 32 \%\end{array}$ \\
\hline $\begin{array}{l}\text { Morales, } \\
\text { Villavicencio } \\
(2018)\end{array}$ & $\begin{array}{l}\text { Cuentas por cobrar y su } \\
\text { relevancia en la liquidez. Art. }\end{array}$ & $\begin{array}{l}\text { Analizar de qué manera } \\
\text { las cuentas por cobrar } \\
\text { afecta en la liquidez }\end{array}$ & $\begin{array}{l}\text { Afectan directamente a la } \\
\text { liquidez de la empresa }\end{array}$ \\
\hline $\begin{array}{l}\text { Morales, } \\
\text { Carhuancho } \\
(2020)\end{array}$ & $\begin{array}{l}\text { Estrategias financieras para } \\
\text { mejorar las cuentas por cobrar } \\
\text { en la Compañía Aquasport } \\
\text { SAC Art. }\end{array}$ & $\begin{array}{l}\text { Proponer estrategias } \\
\text { financieras para mejorar } \\
\text { las cuentas por cobrar }\end{array}$ & $\begin{array}{l}\text { Se reestructura las políticas } \\
\text { de cobranza, debido a que } \\
\text { el total del activo corriente } \\
\text { representa el } 65 \%\end{array}$ \\
\hline $\begin{array}{l}\text { Julca, } \\
\text { Miranda } \\
\text { (2018) }\end{array}$ & $\begin{array}{l}\text { "La gestión de las cuentas por } \\
\text { cobrar y su incidencia en la } \\
\text { liquidez de las pequeñas } \\
\text { empresas": Una revisión } \\
\text { sistemática de la literatura } \\
\text { científica en los últimos } 10 \\
\text { años. Art. }\end{array}$ & $\begin{array}{l}\text { Identificar la relevancia de } \\
\text { la gestión de las cuentas } \\
\text { por cobrar y su incidencia } \\
\text { en las pequeñas empresas }\end{array}$ & $\begin{array}{l}\text { Se evidencia en el estudio } \\
\text { que las empresas en } \\
\text { América Latina tienen una } \\
\text { idea clara sobre la gestión } \\
\text { de cuentas por cobra y } \\
\text { como incide en la liquidez. }\end{array}$ \\
\hline
\end{tabular}

Fuente: Diaz, Flores 2019

Los antecedentes analizados avalan que el presente artículo de revisión es relevante por el impacto de una adecuada gestión de cobranza para disminuir la morosidad y generar liquidez. Al respecto Pibaque y Carrasco (2016), en su investigación consideran establecer una viabilidad en la las políticas y procedimientos de cobranzas para un efectivo control en el pago.

En este contexto el presente artículo cubrirá el espacio vacío que existe en el ámbito financiero, de tal modo que, se pueda corregir deficiencias en las cobranzas, evitar el crecimiento de las deudas, para garantizar a las empresas el retorno económico a corto plazo para cumplir con sus obligaciones.

El objetivo de este artículo es determinar de qué manera una buena gestión de cobranza afecta favorablemente a las empresas, y de esta manera mejorar la liquidez del activo circulante. Es por eso, que Carrera (2017) nos recalca la importancia de la revisión de las políticas de créditos, los objetivos, estrategias utilizadas; mediante las diversas técnicas aplicadas que permita revisar y analizar cada uno de los objetos mencionados anteriormente. 


\section{MATERIALES Y METODOS}

Según Sampieri, Fernámdez, y Baptista, (2014), la investigación documental es detectar, obtener y consultar la biografía y otros materiales que parten de otros conocimientos $\mathrm{y} / \mathrm{o}$ informaciones recogidas moderadamente de cualquier realidad, de manera selectiva, de modo que puedan ser útiles para los propósitos del estudio. El presente trabajo de revisión está basado en una investigación de estudio descriptivo, de tipo documental, teniendo en cuenta que se ha empleado la búsqueda y recopilación de documentos, centrándose en analizar información a profundidad sobre una variable.

Por lo que se puede mencionar, que en la investigación de la variable de cuentas por cobrar, se aplicó el diseño de revisión documental mediante la técnica de análisis sistemático de información a partir de estudios desarrollados en referencia a la temática. Previo al análisis se hizo una búsqueda en base de datos especializada; para la búsqueda a Concytec y como motor de búsqueda a Scielo; como base de datos multidisciplinaria se eligieron Google Académico, como buscador genérico se empleó Redalyc, seleccionando 20 trabajos originales entre artículos y tesis (5). El análisis se centró en los conceptos y planteamientos teóricos sobre la variable descrita.

\section{Proceso de recolección de información}

En ese sentido, para la recopilación de fuentes se han usado de tipo primarias como secundarias; las cuales fueron evaluadas previamente para el desarrollo de la revisión; con respaldos bibliográficos y soporte de autores reflejados mediante las citas referenciales. Finalmente, una vez obtenido los documentos adecuados se han realizado el desarrollo de la revisión que ha buscado ir en concordancia con el objetivo de la investigación para recaer en las conclusiones correctas.

Para garantizar la veracidad del proceso de búsquedas se buscó las variables: Cuentas por Cobrar, La Gestión de las Cuentas por Cobrar, Morosidad y Liquidez. Para especificar la búsqueda de las variables, se realizó la combinación de los términos establecidos y los operadores booleanos: [("Las Cuentas por Cobrar" OR "Incidencia en la Liquidez ") AND ("Las Cuentas por Cobrar y su Incidencia en la Liquidez") OR ("Las Cuentas por Cobrar en las Pequeñas Empresas") AND ("La Gestión de las Cuentas por Cobrar" OR "La Liquidez en las Pequeñas Empresas")].

Se estudió una población conformada por todos los portales web a través de los siguientes pasos: en Redalyc se analizó la literatura con la búsqueda de la variable gestión de cobranza y cuentas por cobrar, se accedió a artículos desde el periodo 2015 - 2019. En Scielo se trabajó no con conceptos sino con modelos teóricos. En Google Académico, se hizo búsqueda por títulos. Mediante Concytec se encontró también teoría de artículos empíricos con datos que recogieron en su investigación, ya que en estos artículos se trabaja con resultados, etc. por tal motivo, se tomó la información de la Introducción.

Asimismo, dentro del estudio también se tomó 5 tesis las que fueron buscadas en Concytec. Los artículos que se escogieron fueron importados haciendo uso de gestores bibliográficos como Mendeley con el propósito de evitar la duplicidad de citas textuales. 
Leydy Diaz y Daibis Yesica Flores Enríquez

\section{Las rutas específicas de búsqueda se describen a continuación:}

Scielo: https://scielo.org/

Google Académico: https://scholar.google.es/

Redalyc: https://www.redalyc.org/

Concytec: http://portal.concytec.gob.pe/

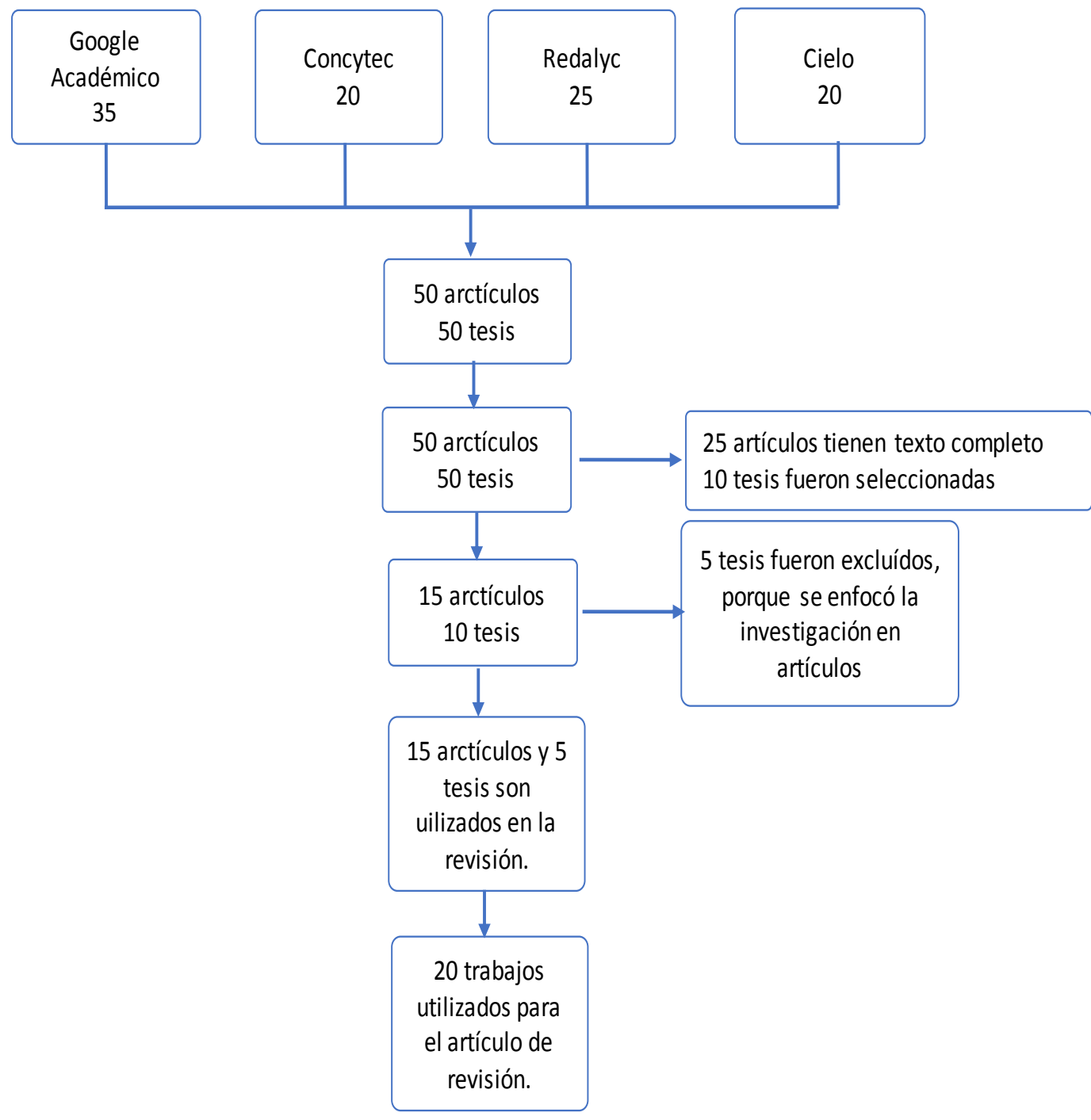

Figura 1. Diagrama de Gestión de cobranza en función a la búsqueda de información de acuerdo a la metodología.. Fuente: Díaz, Flores 2019 


\section{RESULTADOS Y DISCUSION}

Seguidamente, se demuestra el desarrollo de la revisión de la literatura considerando en primer lugar, el fundamento teórico de las cuentas por cobrar y gestión de cobranza. En segundo lugar, la importancia de la gestión de cobranza. En tercer lugar, tipos de gestión de cobranza. En cuarto lugar, las teorías de gestión de cobranza. En quinto lugar, se detalla la metodología de la investigación. En sexto lugar, las conclusiones.

\section{a. Cuentas por cobrar}

Cabrera y Chañi (2019) definen las cuentas por cobrar como un derecho de cobro a corto plazo que tiene a favor una organización cuando haya efectuado una venta a crédito, al cliente por un servicio o en bienes, son parte del activo exigible el cual generan una utilidad en el futuro. Estas provienen cuando un cliente se compromete en pagar por el servicio prestado o los bienes en una fecha establecida, ya que muchas veces llegan a ser incobrables, afectando la utilidad de la organización y se refleja como un activo circulante.

Tanaka (2016) refiere que las cuentas por cobrar son retribuciones obtenidas por la empresa en procedimientos de venta y prestación de servicios realizados al crédito con terceros y relacionados según sea el giro del negocio, para ello los comprobantes de la operación o el contrato realizado será el sustento de la existencia de estos derechos exigibles (Morales y Morales, 2014), estas cuentas se representaran en el Estado de Situación Financiera según sea la fecha en que se realice el cobro (Herz, 2015).

Gonzales y Sanabria (2016) menciona que las cuentas por cobrar más representativos en las partidas del activo es a los clientes vencidas concernientes a productos vendidos o servicios prestados. No es de extrañar que las cuentas de clientes reflejen entre el $30 \%$ y el $50 \%$ de los activos circulantes de la organización. Orihuela (2017) menciona que para mejorar el incremento de ingresos y rentabilidad en la empresa, es importante llevar un seguimiento e inspección de las cobranzas en forma constante, de igual forma llevar un registro del mismo ya que al ser parte de los activos se debe verificar para buscar una liquidez efectiva. Burga y Rodrigo (2019) concluye que es necesario que el área de créditos y cobranzas lleve una buena inspección de los compromisos que tienen los clientes con la organización.

Nova (2008) las cuentas por cobrar, representan los derechos a reclamar efectivo o bienes y servicios como consecuencia de las ventas de mercancías, prestación de servicios, préstamos y otras operaciones a crédito. Bravo (2010) indica que las cuentas por cobrar simboliza un derecho, que se origina por la venta o por los servicios prestados a créditos, la que permite a las empresas obtener mayor liquidez de manera solvente sin problemas financieros y logre el incremento de la rentabilidad.

\section{b. Gestión de cobranza}

Según los fundamentos de la administración, Robles (2012), define como un conjunto de acciones coordinadas, siempre en cuando se aplique adecuadamente y en el momento preciso, de tal modo se consiga recuperar el crédito otorgado a los clientes que no cumplen en pagar por la que se les denomina morosos; al recuperar la cartera se logrará que los activos exigibles lleguen a convertirse en activos líquidos. Asimismo, es 
importante cuidar del cliente para que tenga interés y disposición para futuras negociaciones. Por otro lado Pacheco (2012) indica que son procesos para una adecuada cobranza al vencimiento de los créditos, dichos procedimientos y políticas deben ser eficientes, la que se verá evidenciado en el nivel de gastos por cuentas incobrables. Asimismo, Sala (2015) indica que el objetivo de la gestión de cobranza es de crear una relación comercial con los clientes, de tal manera estos se mantengan al día en el pago aprovechando la ventaja de los productos.

Según las definiciones representadas se puede definir también "como procesos y procedimientos que una empresa puntualiza con la finalidad de cobrar sus servicios prestados a sus clientes para recuperar su liquidez del crédito concedido y al mismo tiempo mantener una buena relación productiva con el cliente".

Lizárraga (2010) concluye que una competente gestión de las cuentas por cobrar representa estar incorporada a un programa de tecnología bancaria, para poder suscribir al financiamiento de las ventas, optimizar el incremento del efectivo y eficiencia con que una empresa cobra sus facturas pendientes. (Cabrera y Chañi, 2019) cuando una empresa no cuenta con límites de crédito (llamadas también líneas de crédito) ésta se expone a aumentar los riesgos de morosidad, pues, justamente un límite de crédito estudiado técnicamente sirve para medir la capacidad de pago de un cliente y en función de este estudio es que la empresa disminuye sus riesgos.

Para Flores (2017) La liquidez representa a los activos que pueden convertirse en dinero de manera inmediata. También representa la capacidad económica que tiene la empresa para hacer frente sus obligaciones a corto plazo. En cierto nivel le permitirá a la empresa disminuir el grado de incertidumbre asociada a la posibilidad de declararse insolvente.

Haro y Rosario (2017) mencionan que la liquidez es la tenencia de activos que puedan convertirse en el corto plazo en efectivo, de tal manera que en el momento oportuno nos permita hacer el pago de los compromisos anteriormente contraídos. (Rodríguez, 2017) la liquidez es el conjunto de aquellos recursos guardados, que pueden usarse como efectivo en cualquier momento. Se trata de fondos destinados a necesidades de corto plazo que requieren un flujo de libre acceso.

\section{c. Importancia de la gestión de cobranza}

La economía capitalista está basado en la acumulación y parte consustancial de esto es la financiación, por tanto todo sabio empresario recurre a este mecanismo para mejorar la acumulación con la mayor rapidez posible. El financiamiento genera el crédito, que puede volverse en contra del espiritu emprendedor si no se tiene apropiadas políticas de conversión en efectivo, en este proceder reside su importancia de gestión.

El término de gestión de cobranza se ha tornado importante debido a la colocación de productos y servicios a través de las ventas a crédito; por lo tanto, la disponibilidad de liquidez para el capital de trabajo de la empresa depende de una gestión eficaz de la cobranza.

De acuerdo con (Aguilar y Remy, 2018) la importancia de la gestión de cobranza es disponer a resolver problemas empresariales en la vida cotidiana y a controlar situaciones que se encuentran normalmente en las empresas; actualmente este tipo de 
investigación se posiciona como un ámbito muy importante, ya que estos estudios de investigación aplicada que se centra en la resolución de problemas en un contexto determinado, es decir, busca la aplicación o utilización de conocimientos, desde una o varias áreas especializadas, con el propósito de implementarlos de forma práctica para satisfacer necesidades concretas, proporcionando una solución a problemas del sector social o productivo. Además, Ettinger citado por Nole, (2015) define que los procedimientos para la cobranza revisten gran importancia, debido a que capacitan a la acreedora para reinvertir su capital, mantener el volumen de las ventas y desarrollar hábitos de pago puntual, en los clientes.

Tumbaco (2017) mencionan que es muy necesario hoy en día aumentar las ventas y las empresas se sienten obligados a implementar políticas de ventas al crédito de esta manera podemos argumentar que las cuentas por cobrar es igual que cualquier activo, es un recurso económico de propiedad de una empresa que le generan un beneficio en el futuro. Las políticas de crédito: son los criterios que aplica la empresa para determinar si debe conceder el crédito a un cliente y las condiciones de otorgamiento, el criterio mínimo para conceder el crédito a un cliente. Cuestiones como periodos promedio de pago, evaluaciones de crédito y referencias Estos criterios financieros brindan una base cuantitativa para establecer y hacer ejecutar las políticas de crédito.

\section{d. Tipos de gestión de cobranza}

Gomez (2012) indica que las etapas de gestión de cobro son las siguientes:
Primero. Preventiva: La empresa efectuará con anterioridad a la fecha de vencimiento la gestión, con la finalidad de recordar la fecha de vencimiento del próximo pago.

Segundo. Administrativa: LoS asesores encargados de la cartera realizan las gestiones desde el primer día de mora, para recuperar el capital e interés generados por el crédito otorgado.

Tercero. Pre jurídico: Con la finalidad de recuperar la cartera en mora, es decir la deuda de los clientes, la empresa realiza la actividad o contrata a terceros que están autorizados por la Caja para recuperar la cartera en mora.

Cuarto. Judicial: Se realiza al no lograrse la recuperación de la cartera, la empresa empieza las cobranzas judiciales mediante abogados internos, los que son cubiertos por el cliente moroso.

\section{e. Teorías de la gestión de cobranza}

Sobre las teorías de gestión de cobranza se identificaron las siguientes definiciones. La gestión de cobranza es la recuperación de la totalidad de la cartera de la empresa donde el departamento de cobranza es responsable de recuperar las cuentas y documentos por cobrar en su totalidad y no permitir que sus finanzas sufren alguna pérdida y que tiene por objetivo cerrar el círculo de la venta (Molina, 2005)

Asimismo, la gestión de cobranza radica en el desarrollo de las actividades y estrategias para lograr el cobro de deudas, para que una gestión de cobranza sea positiva, se debe tomar en cuenta el entorno de negocio y la comunicación; todos estos puntos originan que la información sea bien administrada y 
que se canalice rápidamente para producir efectividad y que en cada gestión la empresa pueda emprender.(Aznar, 2002, p.47)

Asimismo, se define que la Gestión de cobranza, son responsabilidades administrativas y financieras, que están enfocados en captar recursos económicos que proceden de las transacciones económicas o mercantiles. En consecuencia la Gestión de cobros tiene como labor principal obtener la recaudación en una institución (Munch, 2009, p.189)

\section{f. Políticas de cobranza}

Atoche (2016) refiere que, son destrezas que una empresa debe obtener con la finalidad de recuperar o cobrar los créditos que son otorgados a los clientes, siguiendo los procesos de la recuperación, mediante un análisis crediticio, el volumen de las ventas y el promedio de pagos. Gonzáles y Vera (2013) es una ayuda que cada organización utiliza para el mejoramiento de sus cobranzas.

Cárdenas y Blanca (2014) argumentan que las políticas de recuperar el crédito, o de las cuentas por cobrar, son medidas que la dirección de la empresa establece y derivan de la estrategia de riesgos establecida; es decir son medidas que las organizaciones establecen para prevenir riesgos económicos.

Por otro lado Cárdenas y Velasco (2014) mencionan que mediante la ejecución a corto plazo de la políticas de cobranza, hará que se fortalezca la fuerza financiera de la empresa, optimizando la liquidez y el control efectivo y eficiente del costo de las ventas y los gastos de administrativos.

Delgado y Lezama (2015) señalan que para reducir al máximo las pérdidas por las cuentas incobrables, se debe monitorear constantemente aplicaciones de las políticas de cobranza; así el proceso de cobro demostrará firmeza.

\section{g. Liquidez}

Flores (2017) La liquidez representa a los activos que pueden convertirse en dinero de manera inmediata. También representa la capacidad que posee la empresa para enfrentar sus obligaciones en corto plazo. En cierto nivel esto permite disminuir a la empresa el grado de incertidumbre asociada a la posibilidad de declararse insolvente.

Haro y Rosario (2017) la liquidez tener el efectivo necesario, en el momento preciso para que mediante ello se pueda realice el pago de los compromisos anteriormente adquiridos. Rodríguez (2017) la liquidez es el conjunto de aquellos recursos guardados, que pueden usarse como efectivo en cualquier momento. Se trata de fondos destinados a necesidades de corto plazo que requieren un flujo de libre acceso.

Silva (2005) menciona que la liquidez es un sistema, en la visión moderna del neopatrimonialismo, mediante ello las empresas podrán satisfacer necesidades específicas de pago. Por lo tanto, el propósito de la liquidez es promover solvencia al capital. Esta herramienta es indispensable para poder ejecutar actividades administrativas y productivas que las empresas desarrollan a diario.

Gutiérrez, Téllez, y Munilla, (2005) indica que la liquidez de una empresa se valora, con el cumplimiento de pago de las obligaciones a corto plazo según estas hayan vencido. En efecto no sólo se refiere a la cantidad de efectivo disponible, sino como el administrador utiliza sus habilidades para convertir los activos circulantes en flujo de efectivo en el corto plazo y cumplir debidamente las obligaciones negociadas. 
Balarezo (2019) en sus resultados manifiesta que algunas empresas cubren sus obligaciones pero esto les causa limitaciones, y los proveedores por querer cerrar el mes y cumplir su meta ofrecen plazos para cobrar la mercadería que luego no se cumplen y cobran en fechas antes a la negociada, lo cual genera problemas para despachar la siguiente venta, causa retrasos $y$ en muchas ocasiones imposibilita la venta.

\section{CONCLUSIONES}

De acuerdo al objetivo, sobre determinar de qué manera una buena gestión de cobranza afecta favorablemente a las empresas; los estudios analizados muestran que las empresas no son eficientes en la gestión de cuentas por cobrar, ya que el personal a cargo no está cumpliendo con las políticas establecidas por la empresa para poder lograr buenos resultados en la gestión, con respecto a los créditos, la empresa no realiza una revisión minuciosa a los clientes antes de otorgarle el crédito, siendo este el motivo principal para el posterior endeudamiento, otro error de la empresa es otorgar una línea de Crédito abierta sin tener límite ni restricción. Las empresas no realizan un seguimiento que permita recuperar el efectivo de clientes morosos, pero las empresas actualmente no cumplen con este criterio porque sigue incumpliendo las políticas de crédito y cobranza.

Por tanto, con base en la revisión sobre la gestión de cobranza, esta es indispensable para mejorar las finanzas, teniendo en cuenta que al gestionar eficientemente durante el periodo de crédito y la ejecución del pago permite generar flujo de efectivo a la empresa; con ello el incremento de capital de trabajo y liquidez. De esta manera, las empresas se verán favorecidas para seguir invirtiendo en nuevos proyectos y mantener con solvencia para enfrentar sus obligaciones con terceros.

Se considera que la óptima gestión de las cuentas por cobrar tiene incidencia en la liquidez de las empresas; así mismo está vinculada directamente con la respuesta que pueden tener ante las obligaciones financieras, también cabe señalar que la gestión de las cuentas por cobrar en las empresas se deben realizar teniendo en cuenta variables internas; políticas crediticias, administración financiera, etc. que permitirá a la empresa detectar pagos fuera de tiempo, y de esta manera ayudar a reducir las deudas incobrables que pueden ser perjudiciales para las utilidades de la entidad.

Es importante cuidar y fidelizar a los clientes mediante políticas establecidas en cuanto a la concesión de créditos y una buena gestión, ya que por medio de ellos convierten la mercancía en dinero y así se genera el ciclo de vida de una empresa.

\section{REFERENCIAS}

Aguilar, M., \& Remy, C. (2018). Las cuentas por cobrar y su incidencia en la liquidez, en una pequeña empresa. Universidad Privada del Norte

Atoche, L. (2016). Las políticas de crédito y cobranza y su incidencia en la situación económica y financiera de la empresa Despachos Aduaneros Chavimochic S.A.C. del distrito Salaverry, Año 2015. Universidad César Vallejo

Aznar, V. E. M. (2002). El Gestor de Cobranza. Retrieved from https://books.google.com.pe/books?id=ld3 7mei83AIC

Balarezo, M. (2019). Cuentas por cobrar y la liquidez en la empresa Margekal S.A., Cantón Salinas, provincia de Santa Elena, 
año 2018. Universidad Estatal Península de Santa Elena

Bravo, M. de la L. (2010). Introducción a las finanzas (4a. ed.). (4th ed.). Retrieved from https://books.google.com.pe/books?id=6C cttAEACAAJ

Burga, H., \& Rodrigo, S. (2019). Cuentas por cobrar y su incidencia en los Indicadores de liquidez del Centro de Aplicación Imprenta Unión, Lima, Periodos 2013-2017. Universidad Peruana Unión, Lima

Cabrera, Y., \& Chañi, L. (2019). Cuentas por cobrar y su efecto en la liquidez de la Asociación Educativa Adventista Sur Oriental del Perú, periodos 2014 - 2018. Universidad Peruana Unión, Lima

Cárdenas, G., \& Velasco, M. (2014). Incidencia de la morosidad de las cuentas por cobrar en la rentabilidad y la liquidez estudio de caso de una Empresa Social del Estado prestadora de servicios de salud / The incidence of delinquent accounts receivable in profitability and liquidity a case. Revista Facultad Nacional de Salud Pública, 32(1), 16-25

Carrera, S. (2017). "Análisis de la Gestión de cuentas por cobrar en la empresa Induplasma S.A. en el año 2015." Universidad Politécnica Salesiana Ecuador, Guayaquil

Castillo, M., \& Córdova, R. (2016). Gestión de Créditos y cobranzas y su influencia en el nivel de morosidad. Universidad Inca Garcilaso de La Vega

Delgado, C., \& Lezama, N. (2015). Políticas de cobranza para reducir el índice de morosidad. Universidad Privada Antenor Orrego. https://doi.org/10.1007/s001010050327

Díaz, L., \& Ramos, V. (2018). Indicencia de las Políticas de Cobranza en la Liquidez

Flores, A. (2017). Cuentas por cobrar y su relación con la liquidez de las instituciones educativas, distrito de La Molina, año 2017. Universidad César Vallejo, Lima

Flores, M., \& Naval, Y. (2016). Gestión de cuentas por cobrar y la rentabilidad de las empresas del sector industrial que cotizan en la Bolsa de Valores de Lima, períodos 2010 al 2014. Revista de Investigación Universitaria.

https://doi.org/https://doi.org/10.17162/riu .v6i1.1039 Volumen

Gomez, G. Política de cobro. , (2012)

Gonzáles, A., \& Vera, E. (2013). Incidencia de la gestión de las cuentas por cobrar en la liquidez y rentabilidad de la empresa Hierros San Feliz CA, 2011. Universidad Nacional Expiremental de Guayana

Gonzales, E., \& Sanabria, S. (2016). Gestión de cuentas por cobrar y sus efectos en la liquidez en la facultad de una universidad particular, Lima, periodo 2010- 2015. Universidad Peruana Unión, Lima

Gutiérrez, M., Téllez, L., \& Munilla, F. (2005). La Liquidez Empresarial y su Relación con el Sistema Financiero. Ciencias Holguín, XI, 110

Haro, A., \& Rosario, J. (2017). Gestión Financiera: Decisiones financieras a corto plazo. Retrieved from https://books.google.com.pe/books?id=MX QrDWAAQBAJ

Herz, J. (2015). La importancia de la contabilidad. In Apuntes de contabilidad financiera (2nd ed., pp. 1-18). https://doi.org/10.19083/978-612-4191-725

Lizárraga, K. (2010). Gestión de las cuentas por cobrar en la administración del capital de trabajo de las empresas de fabricación de plástico del distrito de Ate. Universidad San Martín de Porres, Lima

Molina, V. (2005). Estrategias de Cobranza en Época de Crisis. Retrieved from https://books.google.com.pe/books?id=Fi M8HWIZWzsC

Morales, A., \& Morales, J. (2014). Crédito Y Cobranza (México; P. edición Ebook, Ed.). Grupo Editorial Patria

Munch, L. (2009). Administracion. Retrieved from

https://books.google.com.pe/books?id=oH rkSAAACAAJ 
Nole, G. (2015). Estrategias de cobranza y su relación con la morosidad de pagos de servicios en la Zonal Ferreñafe de la entidad prestadora de servicios de saneamiento de Lambayeque S.A 2015. Universidad Señor de Sipán

Nova, A. (2008). Finanzas para no financistas (4th ed.). Retrieved from https://books.google.com.pe/books?id=Jm C1zekAM7oC

Orihuela, J. (2017). Gestión de cuentas por cobrar y su relación con el estado de resultados de las instituciones educativas particulares de educación básica regular del distrito de Villa María del Triunfo, 2017. Universidad César Vallejo, Lima

Pacheco, J. (2012). Gestión de cobranzas con Excel (primera ed; Editorial Macro, Ed.). Retrieved from https://books.google.com.pe/books?id=un 4vDgAAQBAJ

Pibaque, I., \& Carrasco, R. (2016). Análisis de las políticas y procedimientos de cobranza - Unidad Educativa José María Urbina. Universidad de Guayaquil

Robles, C. (2012). Fundamentos de administración financiera. In Fundamentos de administración financiera (1st ed.). https://doi.org/10.1016/S00223476(05)80868-6

Rodríguez, S. (2017). Finanzas personales su mejor plan de vida (Uniandes,). Retrieved from
https://books.google.com.pe/books?id=dZ FcDwAAQBAJ

Ruiz, A., \& Lopez, J. (2017). Influencia de la Gestión de Cobranza en la Reducción de la Morosidad

Sala, G. (2015). Gestión de un pequeño comercio (Editex, Ed.). Retrieved from https://books.google.com.pe/books?id=b7JCQAAQBAJ

Sampieri, R., Fernámdez, C., \& Baptista, P. (2014). Metodología de la investigación (M.-H. Education, Ed.). Cámara Nacional de la Industria Editorial Mexicana

Santillán, J. (2016). Las Cuentas por cobrar y su impacto en la liquidez de la empresa importadora industrial agrícola S.A. IIASA. Universidad Laica VICENTE ROCAFUERTE de Guayaquil

Silva, R. A. C. (2005). Filosofia da Análise da Estabilidade da Liquidez. Revista Catarinense Da Ciência Contábil, 4(11), 51$64 . \quad$ https://doi.org/10.16930/22377662/rccc.v4n11p51-64

Tanaka, G. (2016). Contabilidad y análisis financiero: Un enfoque para el Perú (Fondo Edit). Retrieved from https://books.google.com.pe/books?id=N6 LNDWAAQBAJ

Tumbaco, L. (2017). Plan estratégico para la gestión de cobranza en Disan Ecuador S.A. Universidad de Guayaquil 